\title{
CW-THz Wave Generation Using a Multimode Semiconductor Laser at Room Temperature
}

\author{
Sylwester Latkowski, Pascal Landais \\ RINCE, School of Electronic Engineering \\ Dublin City University \\ Glasnevin, Dublin 9, Ireland \\ Email: sylwester@eeng.dcu.ie
}

\author{
Frederic Surre \\ Physics Department \\ Trinity College Dublin \\ Dublin 2, Ireland \\ Email: surref@tcd.ie
}

\author{
Stephen A. Lynch \\ London Centre for Nanotechnology \\ University College London \\ London, UK \\ Email: slynch@ucl.uk
}

\begin{abstract}
Multimode semiconductor lasers under certain DC bias conditions generate electromagnetic waves from the beating between the longitudinal lasing modes. The frequency of those waves is directly related to the free spectral range of the involved modes. Our recent experimental work is carried out on slotted Fabry-Perot lasers with mode separation of $330 \mathrm{GHz}$. The devices are at room temperature biased at 3 times the threshold current. Using a bolometer coupled to an FT-IR interferometer we are able to observe radiation at $660 \mathrm{GHz}$, in the terahertz range.
\end{abstract}

\section{INTRODUCTION}

Modes beating in DC biased semiconductor multimode laser generates a fluctuation of the light emission, sometimes referred as self-pulsation (SP) [1]. The frequency of this fluctuation corresponds to the free-spectral range (FSR) or multiples of it.

It has been demonstrated that multimode distributed Bragg reflector (DBR) lasers with a FSR of $40 \mathrm{GHz}$ are able to synchronize its SP frequency to the clock frequency of an external optical data signal [2]. The clock recovery achieved in this matter could be used in all-optical data processing such as in re-amplify, re-time, and re-shape (3R) function or in logical gate. It has been proven and demonstrated that the origin of the SP in these multimode DBR lasers relies on the carrier density pulsation (CDP). This four-wave-mixing (FWM) effect results in the nonlinearity which leads to a passive locking of the phase of the lasing modes demonstrated by the fact that the linewidth of the $40 \mathrm{GHz}$ signal is smaller than the sum of the linewidth of the optical lasing modes [3].

If the detuning between modes increases, can we still assume that FWM effects can contribute to this passive modelocking? Is it possible to generate a $\mathrm{THz}$ signal with a multimode laser? In this presentation, we would like to address these questions. We present and discuss some results achieved with a devices where beating frequency falls into the $\mathrm{THz}$ regime.

\section{DEVICE UNDER TEST AND CHARACTERIZATION}

The device under test is a multi-quantum wells InAlGaAs Fabry-Perot (FP) laser It is a $2.5 \mu \mathrm{m}$ ridge waveguide laser resulting in spatial single mode output. It is $350 \mu \mathrm{m}$ long and the associated spectral range is calculated at $\sim 123 \mathrm{GHz}$ as measured in Fig. 1. By etching grooves at key positions along the top contact of the active layer it is possible to design a one dimensional photonic band-gap. The FP resonance determines the spectral separation between consecutive modes, but the etched band-gap suppresses or enhances some specific modes. It is possible therefore to choose some given modes and to control the detuning between modes, therefore the SP frequency.

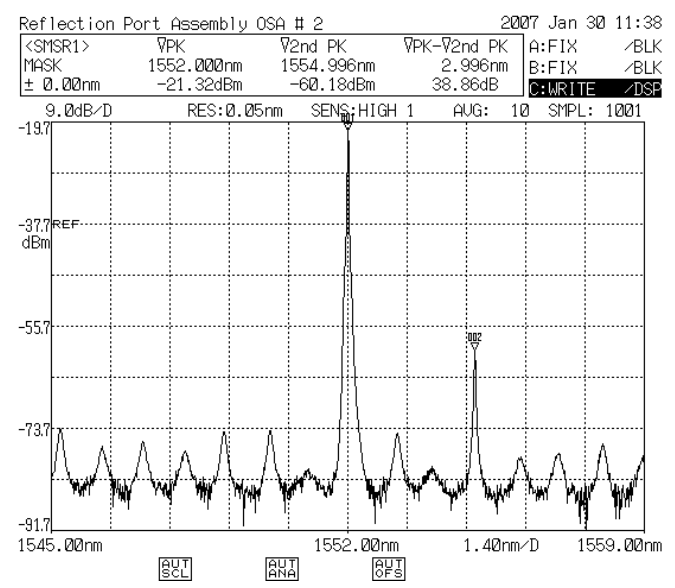

Fig. 1. Optical spectrum of the laser under DC bias at $60 \mathrm{~mA}$ and temperature controlled at $25^{\circ} \mathrm{C}$.

The optical spectrum shown in Fig.1 has been recorded for a bias current of $60 \mathrm{~mA}$, corresponding to three times the threshold current. The laser is temperature controlled at $25^{\circ} \mathrm{C}$. The resolution of the optical spectrum analyzer is $0.05 \mathrm{~nm}$. The longitudinal spectrum features a main mode at $1552.00 \mathrm{~nm}$ and the second mode at $1554.99 \mathrm{~nm}$. The side-mode-suppressionratio is greater than $30 \mathrm{~dB}$ and the device is considered singlemode for telecommunication applications. In the light-current curve (not presented in this document) there is no presence of hysteresis loop, thus the laser cavity does not include a saturable absorber section. The separation between the modes is of $330 \mathrm{GHz}$.

\section{THz EXPERIMENTAL SETUP AND RESULTS}

The $\mathrm{THz}$ signal is investigated using a Fourier-Transform infrared (FT-IR) spectrometer equipped with a bolometer. The 
spectral resolution of this set-up is $0.05 \mathrm{~cm}^{-1}$. The emission of the laser is collimated to the bolometer by a $125 \mu \mathrm{m}$ beamsplitter limiting the detection between 50 and $5 \mathrm{~cm}^{-1}$. Hence it is expected that only the $\mathrm{THz}$ signal is launched into the detector and no optical signal. The beating between the main modes of the device is $330 \mathrm{GHz}$, and this is just below the range of detectivity of the bolometer set-up, thus it was not possible to observe the beating signal with this set-up. However, a THz signal is observed. This spectrum is shown in Fig.2. It features a $\mathrm{THz}$ signal at $0.66 \mathrm{THz}$, with a linewidth of $27 \mathrm{GHz}$. For this measurement, the laser is DC biased $60 \mathrm{~mA}$ and temperature controlled at $25^{\circ} \mathrm{C}$. The signal observed corresponds to second harmonic of the beating. It is well above the noise floor. It is expected that the fundamental mode at $330 \mathrm{GHz}$ has a linwdth smaller than $27 \mathrm{GHz}$.

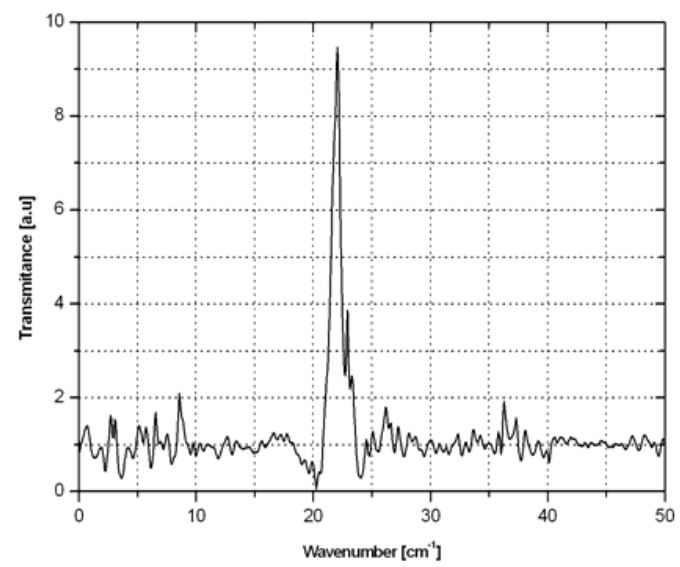

Fig. 2. THz signal measured from device under DC bias at $60 \mathrm{~mA}$ and temperature controlled at $25^{\circ} \mathrm{C}$.

Using a frequency resolved optical gating (FROG) analyser, the output signal of the laser can be analysed in the frequency and in the time domains. The time variation of the second harmonic signal from the two main modes is presented in Fig. 3 . The two main modes are out of phase, varying sinusoidally at a frequency of $370 \mathrm{GHz}$. At this frequency, the time associated with the carrier density pulsation is not fast enough to provide such modulation, therefore the modulation of the signals is produced by intra-band effects, and at this frequency the dominant effect is carrier heating. It is also clear from Fig. 3 that the two modes are synchronized and therefore the carrier heating effect is contributing to the passive modelocking of the two main modes.

\section{CONCLUSIONS}

We have demonstrated the generation of $\mathrm{THz}$ waves from mode beating in a multimode laser. The laser studied is a Fabry-Perot cavity with with slots implemented to control the longitudinal spectrum, which results in two modes separated

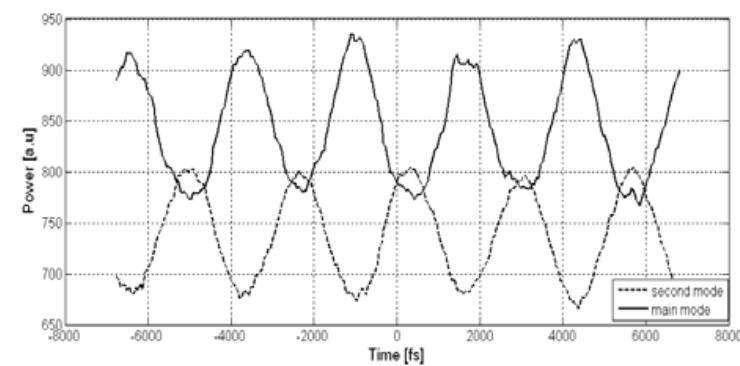

Fig. 3. Time variation of the second harmonic generation of the two main modes.

by $3 \mathrm{~nm}$. Even though the laser is DC biased and at room temperature it produces an electromagnetic waves at $370 \mathrm{GHz}$ and $660 \mathrm{GHz}$ corresponding to multiple of the free spectral range. The origin of this modulation relies in carrier heating nonlinear effect and produces a passive synchronization of the optical modes.

\section{REFERENCES}

[1] K. Sato, "100 GHz optical pulse generation using Fabry-Perot laser under continuous wave operation," Electronics Letters, vol. 37, pp. 763-764, 2001.

[2] J. Renaudier, B. Lavigne, P. Gallion, G.-H Duan, "Study of phase-noise properties and timing jitter of $40-\mathrm{GHz}$ all-optical clock recovery using self-pulsating semiconductor lasers, " J. Lightwave Technology, vol. 24, pp. 3734-3742, 2006.

[3] J. Renaudier, G.-H. Duan, P. Landais , P. Gallion , "Phase correlation and linewidth reduction of $40 \mathrm{GHz}$ self-pulsation in distributed Bragg reflector semiconductor lasers, " IEEE J. Quantum Electron., vol. 43, pp. $147-156,2006$ 